\title{
BRAF Mutation Status in Primary, Recurrent, and Metastatic Malignant Melanoma and Its Relation to Histopathological Parameters
}

\author{
Aris Spathis ${ }^{1}$, Alexander C. Katoulis ${ }^{2}$, Vasileia Damaskou ${ }^{1}$, Aikaterini I. Liakou ${ }^{2}$, Christine Kottaridi ${ }^{1}$, \\ Danai Leventakou ${ }^{1}$, Dimitrios Sgouros ${ }^{2}$, Andreas Mamantopoulos ${ }^{3}$, Dimitrios Rigopoulos ${ }^{2}$, \\ Petros Karakitsos ${ }^{4}$, Ioannis G. Panayiotides ${ }^{1}$ \\ 1 Second Department of Pathology, National and Kapodistrian University of Athens, School of Medicine, Attikon University Hospital, Athens, \\ Greece \\ 2 Second Department of Dermatology and Venereology, National and Kapodistrian University of Athens, School of Medicine, Attikon \\ University Hospital, Athens, Greece \\ 3 Department of Surgery, Ierapetra General Hospital, Ierapetra (Crete), Greece \\ 4 Department of Cytopathology, National and Kapodistrian University of Athens, School of Medicine, Attikon University Hospital, Athens, \\ Greece
}

Key words: malignant melanoma, histopathological features, $B R A F$ mutations

Citation: Spathis A, Katoulis AC, Damaskou V, Liakou AI, Kottaridi C, Leventakou D, Sgouros D, Mamantopoulos A, Rigopoulos D, Karakitsos P, Panayiotides IG. BRAF mutation status in primary, recurrent, and metastatic malignant melanoma and its relation to histopathological parameters. Dermatol Pract Concept. 2019;9(1):54-62. DOI: https://doi.org/10.5826/dpc.0901a13

Published: January 31, 2019

Copyright: $@ 2019$ Spathis et al. This is an open-access article distributed under the terms of the Creative Commons Attribution License, which permits unrestricted use, distribution, and reproduction in any medium, provided the original author and source are credited.

Funding: None.

Competing interests: The authors have no conflicts of interest to disclose.

Authorship: The first 3 authors contributed equally to this work. All authors have contributed significantly to this publication.

Corresponding author: A. Spathis, PhD, SCYM(ASCP $)^{\mathrm{CM}}$, Second Department of Pathology, National and Kapodistrian University of Athens, Attikon University Hospital, 1, Rimini str., GR-12462 Chaidari, Athens, Greece. Email: aspathis@med.uoa.gr

ABSTRACT Background: BRAF mutations are a common finding in malignant melanoma (MM). Nevertheless, apart from their significance as a therapeutic target in advanced melanoma, their prognostic value is still debated.

Objective: To assess $B R A F$ mutation status in primary, recurrent, or metastatic MM and its correlations with histopathological findings.

Methods: We analyzed 203 samples from 178 consecutive patients: 129 primary cutaneous MM, 49 metastatic and recurrent MM of unknown primary site, and 25 cases of recurrences or metastases of primary MM. BRAF mutations in exon 15 were identified with real-time polymerase chain reaction and/or direct sequencing or pyrosequencing. Histopathological examination was performed according to standard procedures.

Results: We observed a $42.1 \%$ prevalence of BRAF mutations at codon 600 among our patients, $84 \%$ of whom harbored the V600E mutation. Mutations showed a statistically significant increase in 
ABSTRACT younger patients $(\mathrm{P}=0.011)$, in ulcerated tumors $(\mathrm{P}=0.020)$, and in tumors lacking solar elastosis in adjacent dermis $(\mathrm{P}=0.008)$. Mutations were also more common in male patients, as well as in primary MMs of the torso, and in nonvisceral metastases, however without reaching statistical significance. Logistic regression analysis identified type and ulceration as the only significant predictors of $B R A F$ mutation. The highest frequencies of mutated $B R A F$ were identified in superficial spreading and nodular types, and the lowest in acral lentiginous and lentigo maligna types. In situ MM and primary dermal melanoma displayed intermediate frequencies.

Conclusion: Frequency of mutated $B R A F$ is type-related and correlated with ulceration, a known adverse prognostic factor.

\section{Introduction}

Malignant melanoma (MM) is one of the most common types of cancer in humans, being the fifth most common in men and the sixth in women in the United States [1]. Although MM represents less than $2 \%$ of skin cancer, it accounts for the vast majority of deaths, with increasing incidence worldwide [1]. The underlying etiopathogenetic mechanisms remain so far largely unknown.

Following discovery of $B R A F$ oncogenic mutations in various types of malignancies, a significant pathogenetic role for mutated $B R A F$ has been proposed for MM [2]. To date, more than 30 different mutations in the $B R A F$ gene have been identified. The most frequent, accounting for almost $90 \%$, concerns substitution of thymine by adenine at nucleotide c.1799, leading to valine (V) being substituted by glutamate (E) at codon 600 (p.V600E) in the activation segment of the gene promoting cell survival via the $E R K$ or $M E K$ signaling cascade [3]. Frequency of $B R A F$ mutations varies widely, from more than $80 \%$ in melanomas and nevi, to $1 \%-3 \%$ in lung and $5 \%$ in colorectal carcinoma [2]. Of note, BRAF mutations are not exclusive to MM, having also been found in up to $80 \%$ of benign melanocytic lesions, albeit in limited study populations [4].

Oncogenic mutations of $B R A F$ are mimicking a constantly activated state, resulting in uncontrolled cell growth and proliferation. More recently, oncogenic BRAF mutations have been connected to up-regulated cell proliferation and invasion ability [5]. It has been suggested that BRAF mutations may constitute a tumor progression event rather than an initiating event in MM tumorigenesis, and that other genetic or epigenetic factors are also involved $[6,7]$. The presence of a $B R A F$ mutation in primary MM of stage III has been associated with worse prognosis and also seems to be related to progression-free interval and overall survival [8].

Mutated $B R A F$ constitutes a therapeutic target for patients with advanced MMs. Different $B R A F$ inhibitors have been tested, with significant benefits in terms of increased response rate, progression-free survival, and overall survival [9]. Nevertheless, the prognostic value of $B R A F$ mutations has not been fully assessed, with the only established his- topathological parameters associated with prognosis and survival of MMs being tumor thickness, ulceration, and, more recently, mitotic score at primary site [10].

We assessed the BRAF mutation status among 178 patients with primary, recurrent, or metastatic MM. Our aim was to study the correlations between histopathological findings in primary $\mathrm{MM}$ and the presence of mutated $B R A F$ in a Greek population.

\section{Methods}

\section{Specimens}

A total of 203 consecutive specimens from 178 patients referred for histopathological examination during a 12 -year period (2003-2015) were analyzed (Table 1). These included 129 primary cutaneous MMs (a primary site recurrence was also included in 13 cases and a metastasis in 12 cases), 44 cases of metastatic MM of unknown primary site, and 5 cases of cutaneous recurrence without an initial tumor. The institutional review board of the hospital approved the study, while analysis was performed on anonymized data.

All specimens were fixed in a buffered $10 \%$ formalin solution, processed according to standard protocols, and initially diagnosed by the same experienced pathologist. Another, equally experienced pathologist performed a second, blind evaluation of histopathological diagnosis for the purpose of this study. In case of disagreement, slides were conjointly rereviewed.

\section{BRAF Mutation Analysis}

Five 5 - $\mu$ m-thick sections were obtained from a paraffin block containing a representative tumor area, as assessed in a hematoxylin and eosin-stained section. DNA was extracted with Purelink DNA Mini KIT (Invitrogen, Darmstadt, Germany) according to the manufacturer's instructions. Detection of $B R A F$ p.V600E mutation was performed with the use of 2 molecular beacons in a real-time polymerase chain reaction as previously described [11]. All wild-type and selected mutated samples were sent for verification with direct sequencing of exon 15 of $B R A F$ using previously published primers or were 
Table 1. Sample Types and BRAF Status

\begin{tabular}{|c|c|c|c|c|c|}
\hline & \multicolumn{2}{|c|}{ BRAF Status } & \multirow{2}{*}{ Total } & \multirow{2}{*}{ Mutated (\%) } & \multirow{2}{*}{$\mathbf{P}$} \\
\hline & WT & Mutated & & & \\
\hline Patients & 103 & 75 & 178 & 42.1 & \\
\hline Sample type & 111 & 92 & 203 & 45.3 & \\
\hline Primary melanoma & 77 & 52 & 129 & 40.3 & \multirow{5}{*}{0.300} \\
\hline Metastasis of primary & 6 & 6 & 12 & 50.0 & \\
\hline Metastasis w/o primary & 23 & 21 & 44 & 47.7 & \\
\hline Recurrence of primary & 2 & 11 & 13 & 84.6 & \\
\hline Recurrence w/o primary & 3 & 2 & 5 & 40.0 & \\
\hline $\begin{array}{l}\text { Age, mean }(95 \% \mathrm{CI}) \\
(\mathrm{n}=178)\end{array}$ & $\begin{array}{c}64.6 \\
(61.5-67.6) \\
\end{array}$ & $\begin{array}{c}58.9 \\
(55.6-62.2)\end{array}$ & $\begin{array}{c}62.1 \\
(59.9-64.4)\end{array}$ & & 0.011 \\
\hline \multicolumn{6}{|l|}{$\operatorname{Sex}(n=178)$} \\
\hline Male & 55 & 50 & 105 & 47.6 & \multirow{2}{*}{0.090} \\
\hline Female & 48 & 25 & 73 & 34.2 & \\
\hline
\end{tabular}

$\mathrm{CI}=$ confidence interval; $\mathrm{WT}=$ wild type.

sequenced in-house using a commercially available kit (Therascreen BRAF pyro kit, Qiagen, Düsseldorf, Germany) [11].

\section{Primers and Beacons}

$B R A F$ primers and molecular beacons were designed with Beacon Designer 7 (Premier Biosoft, Palo Alto, CA). Oligos were synthesized by Jena Bioscience $\mathrm{GmbH}$ (Jena, Germany).

\section{Quality Assurance}

Both the Department of Cytopathology and the second Department of Pathology have successfully participated in the European Quality Assurance schemes of the European Society of Pathology for molecular identification of mutations in KRAS, NRAS, and BRAF since 2013 and through 2016.

\section{Statistical Analysis}

Statistical analysis was performed using IBM Statistics SPSS 24 (IBM Corporation, New York, NY) and involved the Fisher exact test for $2 \times 2$ tables, $\chi^{2}$ for trend for $2 \times Y$ tables, MannWhitney test for differences of mean of categories, and Spearman correlation analysis for scale correlations. Binary logistic regression was used for validation of observed differences of $B R A F$ status with all mutations grouped in a single category.

\section{Results}

\section{Demographic and Clinical Data}

One hundred seventy-eight melanoma patients aged 21-95 years (mean 62.1 years) were included in the study. There were 105 men with a mean age of 62.4 years and 73 women with a mean age of 61.4 years. Received samples were monitored to identify repeated samples from the same patients for an average of 70 months (range 1-162 months). Recurrent samples were received within 1 to 32 months (mean 8.5 months), while reported metastases were collected within 0 to 49 months (mean 11.9 months). Metastases were identified in lymph nodes (31/56), gastrointestinal tract (6/56), bone marrow (1/56), lung (1/56), ovaries (2/56), liver (7/56), and skin sites distant from the initial tumor area (8/56). All 18 recurrent specimens concerned cutaneous tumors.

\section{BRAF Mutation Distribution}

$B R A F$ mutations were identified in a total of $92 / 203$ samples $(45.3 \%)$. Analyzing only unique samples, $B R A F$ mutations were identified in $75 / 178$ samples $(42.1 \%)$. Of these 75 samples, $63(84 \%)$ carried the V600E mutation (c. $1799 \mathrm{~T}>\mathrm{A}), 10$ samples $(13.3 \%)$ the $\mathrm{V} 600 \mathrm{~K}$ mutation (c.1799_1800TG>AA), 1 sample (1.3\%) carried the V600R mutation (c.1798_1799GT>AG), and 1 sample (1.3\%) the V600A (c.1799T>C) mutation. The V600K, V600R, and V600A mutations were identified by sequencing only, since the molecular beacons, as expected, could not identify them (Figures 1 and 2).

In 12 patients, samples from both primary site and metastasis were available: in 10/12 (83.3\%) cases both sites had the same genotype, whereas one primary carried a V600E mutation not identified in nodal metastasis, and another was WT in the primary with a V600E mutation in the visceral metastasis.

\section{Correlation of $B R A F$ Status to Epidemiological and Clinical Parameters}

Correlation of $B R A F$ mutation status with epidemiological and clinical parameters is shown in Table 1. Results for 

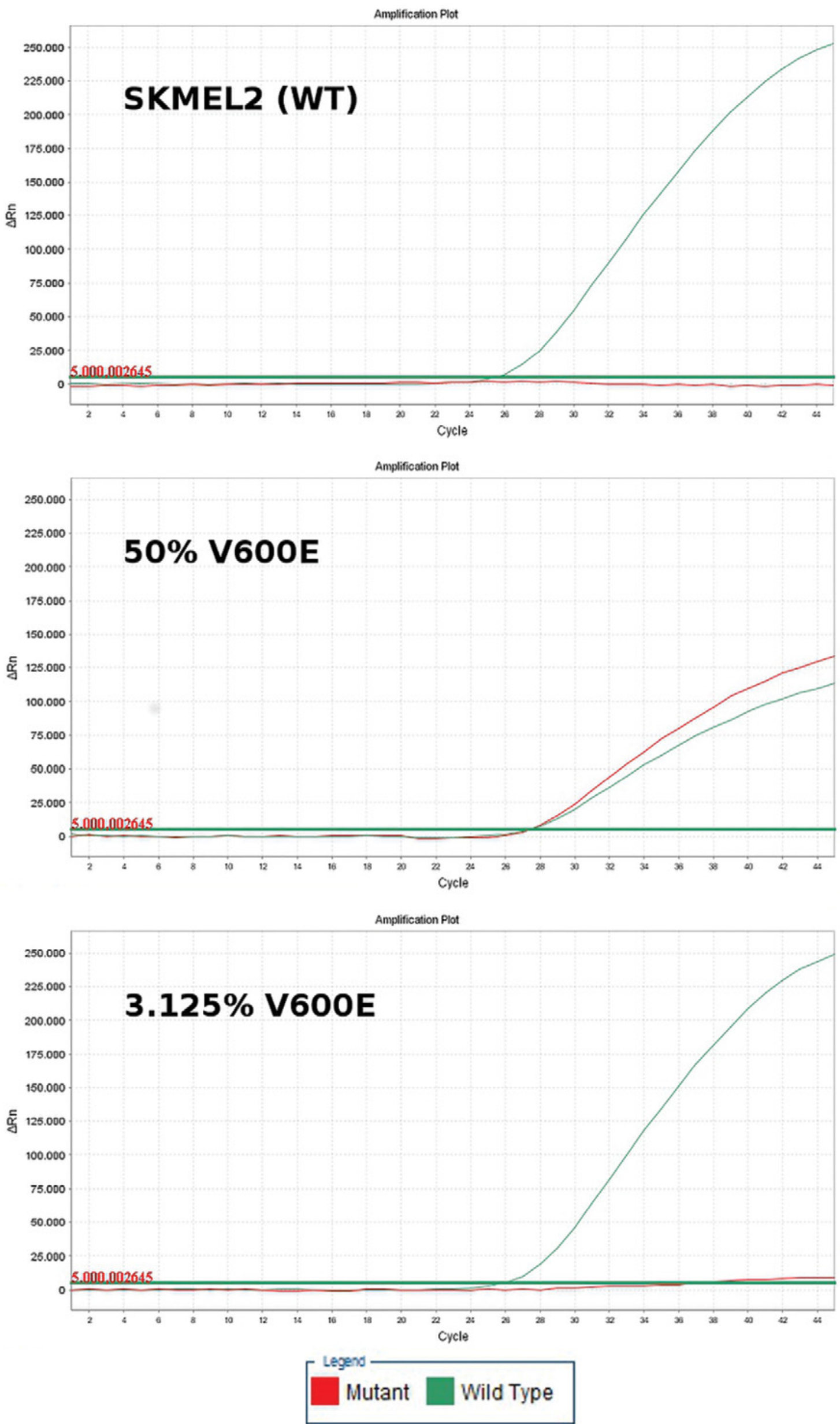

Figure 1. (continues) 
Figure 1, continued
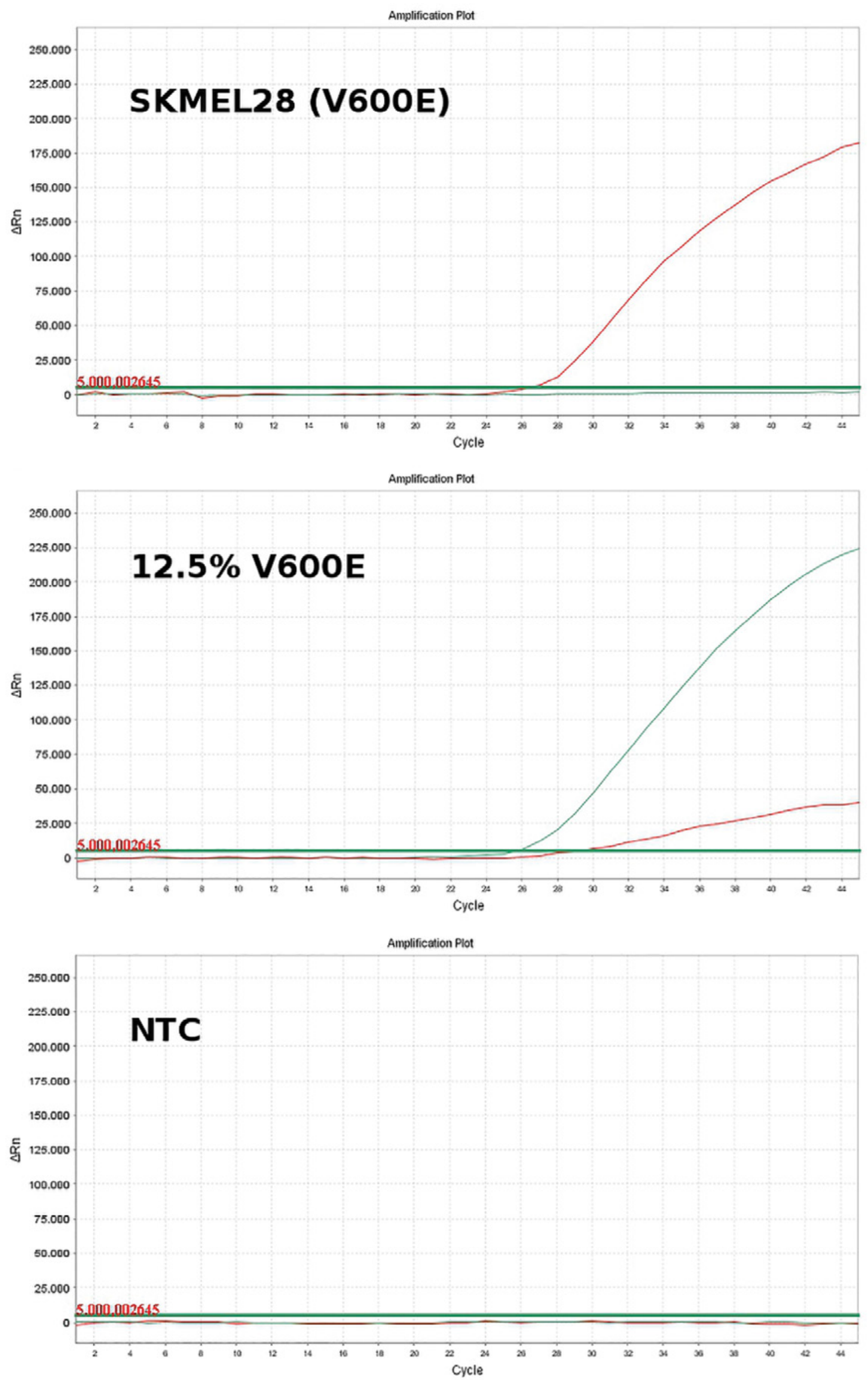

Figure 1. Real-time polymerase chain reaction plots of serial dilutions depicting that even rare mutation-harboring populations be identified using molecular beacons (red-colored line = mutant; green-colored line = wild type). [Copyright: @2019 Spathis et al.] [Copyright: (02019 Spathis et al.] 
WT (GTG)

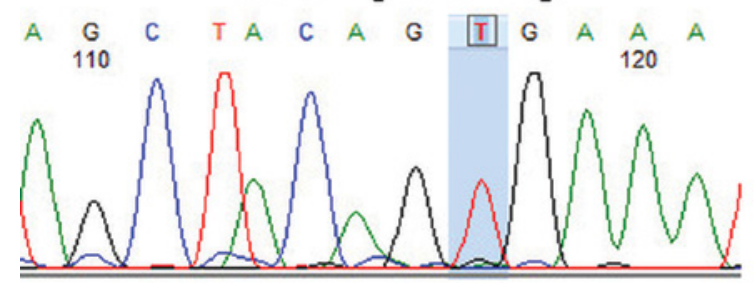

V600K (AAG)

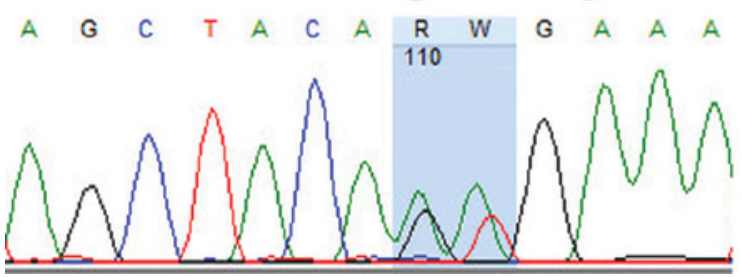

V600E (GAG)

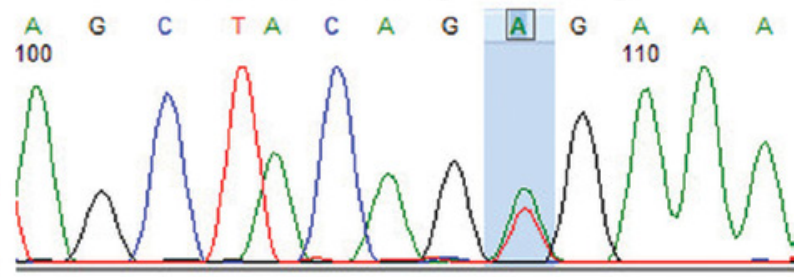

V600R (AGG)

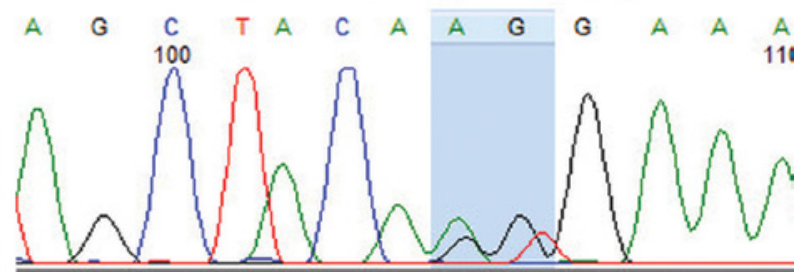

Figure 2. Sequencing chromatographs of a wild-type sample and samples carrying a V600E mutation, a V600K mutation, and a V600R mutation. [Copyright: (C2019 Spathis et al.]

primary and metastatic MMs are shown in Tables 2 and 3, respectively. Briefly, $B R A F$ mutations were more common among younger patients $(\mathrm{P}=0.011$, Mann-Whitney $\mathrm{U}$ test $)$, while the same trend was present for primary and metastatic samples, however without reaching statistical significance. Primary MMs of the torso showed a slightly increased mutation frequency, compared with those located at the extremities or head and neck area $(40.3 \%$ vs $36.3 \%, P=0.269$, Fisher exact test). Cutaneous and lymph node metastases also displayed higher mutation frequency, again without reaching statistical significance $(53.8 \%$ vs $35.2, \mathrm{P}=0.143$, Fisher exact test). Mutations were more common in men, although this was statistically significant only in metastatic samples $(\mathrm{P}=$ 0.029, Fisher exact test).

Patients with a recurrent melanoma had higher mutation frequency $(13 / 18$ vs $41 / 116, \mathrm{P}=0.004$, Fisher exact test), although limited samples were available. Patients with melanoma metastasis showed a slightly increased frequency of $B R A F$ mutations ( $37 \%$ vs $30.7 \%, P=0.413$, Fisher exact test), without reaching statistical significance. These results are only indicative because we analyzed reported metastases and recurrences that had been referred to our laboratory.

\section{Association of BRAF Status to Histopathological Parameters}

In primary MMs (Table 4), mutated $B R A F$ was significantly more common in ulcerated tumors $(51.7 \%$ vs $31 \%, \mathrm{P}=$ 0.002 , Fisher exact test) or when the adjacent dermis lacked solar elastosis $(44.3 \%$ vs $7.1 \%, \mathrm{P}=0.008$, Fisher exact test $)$. In contrast, the number of mitoses, presence of tumor-infiltrating lymphocytes (TILs), Clark level, or Breslow thickness were not significantly related to $B R A F$ mutation status, apart

Table 2. Patient Characteristics of Primary Melanomas and BRAF Status

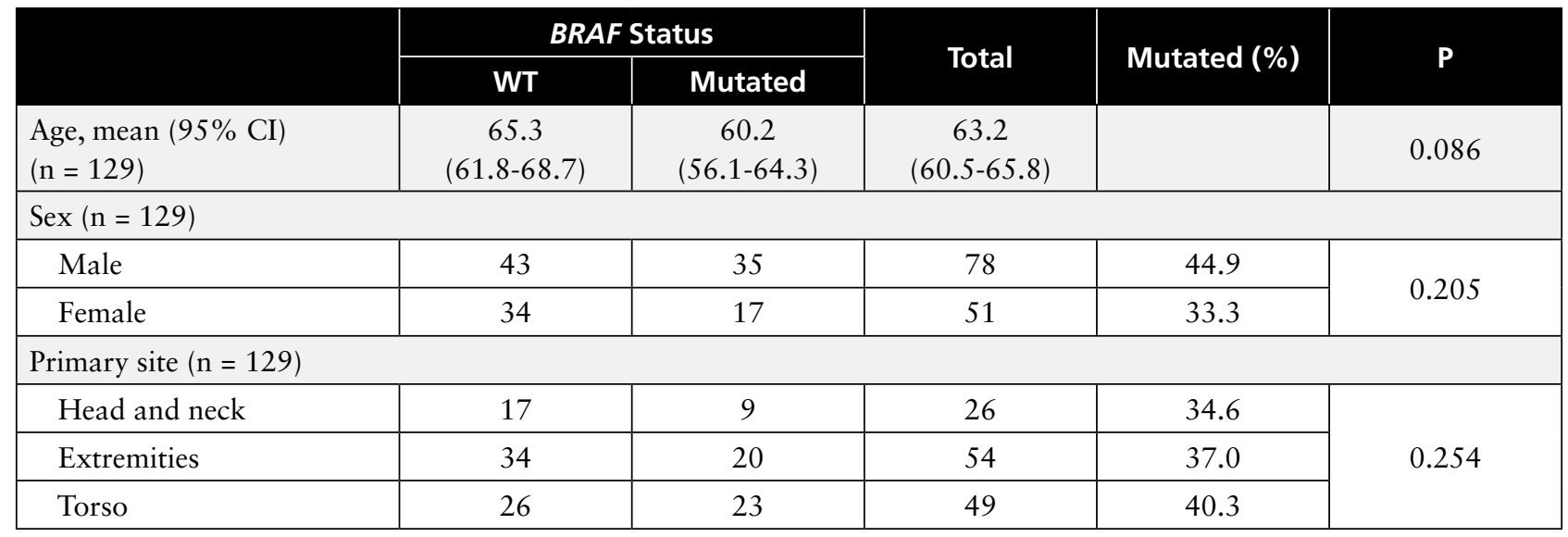

$\mathrm{CI}=$ confidence interval; $\mathrm{WT}=$ wild type. 
Table 3. Patient Characteristics of Metastatic Melanomas and BRAF Status

\begin{tabular}{|c|c|c|c|c|c|}
\hline & \multicolumn{2}{|c|}{ BRAF Status } & \multirow{2}{*}{ Total } & \multirow{2}{*}{ Mutated (\%) } & \multirow{2}{*}{$\mathbf{P}$} \\
\hline & WT & Mutated & & & \\
\hline $\begin{array}{l}\text { Age, mean } \\
(95 \% \text { CI })(\mathrm{n}=56)\end{array}$ & $\begin{array}{c}61.5 \\
(55.3-67.7)\end{array}$ & $\begin{array}{c}57.7 \\
(51.9-63.5)\end{array}$ & $\begin{array}{c}59.7 \\
(55.5-63.8)\end{array}$ & & 0.251 \\
\hline \multicolumn{6}{|l|}{$\operatorname{Sex}(n=56)$} \\
\hline Male & 14 & 21 & 35 & 60.0 & \multirow{2}{*}{0.029} \\
\hline Female & 15 & 6 & 21 & 28.6 & \\
\hline \multicolumn{6}{|l|}{ Metastasis site $(\mathrm{n}=56)$} \\
\hline Distant skin or LN & 18 & 21 & 39 & 53.8 & \multirow{3}{*}{0.149} \\
\hline Lung & 0 & 1 & 1 & 100 & \\
\hline Other visceral & 11 & 5 & 16 & 31.3 & \\
\hline
\end{tabular}

$\mathrm{CI}=$ confidence interval; $\mathrm{LN}=$ lymph node; $\mathrm{WT}=$ wild type

from a slight increase in BRAF mutations among cases with TILs (42.9\% vs $37.5 \%$, Fisher exact test) or MMs with Breslow thickness $>1 \mathrm{~mm}(43.9 \%$ vs $38.1 \%$, Fisher exact test).

Concerning the type of MM, mutations were more common in superficial spreading MM (SSM) and nodular MM (NM) and less common in acral lentiginous MM (ALM) and lentigo maligna melanomas (LMM). Primary dermal MM and intraepidermal (in situ) melanomas displayed an intermediate frequency. The trend was significant when analyzing the different types, whether primary dermal and in situ melanomas were included in the analysis or not. Cross-tabulation of melanoma types with $B R A F$ status divided by presence of ulceration showed similar mutated samples with or without ulceration for ALM (1/4 vs 0/4), LMM (1/5 vs 1/7), and SMM (10/16 vs $13 / 27)$, but not for NM (2/14 vs $18 / 32,14.3 \%$ vs $56.2 \%, \mathrm{P}=0.010$, Fisher exact test).

We then used a backward step-wise Wald binary logistic regression analysis including all borderline and statistically significant parameters. Topology and age were removed during model creation, while ulceration $(\mathrm{P}=0.008$, odds ratio $=$ $3.509)$ and type $(P=0.024)$, specifically SSM type $(P=0.020$, odds ratio $=7.626$ ), were the only predictors.

\section{Discussion}

Per our results, a $42.1 \%$ prevalence of $B R A F$ mutations at codon 600 among our patients with MM was observed. These mutations were statistically more common in younger patients, in ulcerated MMs, and in MMs lacking solar elastosis in adjacent papillary dermis. These findings are consistent with previous experience [7, 12]. A trend for increased percentage of $B R A F$ mutations was documented in men with MMs of the torso, in MMs more than $1 \mathrm{~mm}$ Breslow thickness, higher mitotic rate, reported metastasis, and earlier reported recurrences. However, these findings did not reach statistical significance, as some of them did in previous studies $[12,13]$. Almost all pairs of primary and metastatic sites had the same genotype, except 2 patients, in whom a discordance of $B R A F$ status between primary site and metastasis was found [7, 14].

In a recent meta-analysis, BRAF and NRAS mutations were associated with histological subtype and tumor site, but not with patient age or sex [15]. BRAF mutations were frequently detected in patients with SSM and in MMs arising in non-chronically sun-damaged skin. In contrast, NRAS mutations were more frequent in patients with $\mathrm{NM}$ and in MMs arising in chronically sun-damaged skin [15]. Another meta-analysis of BRAF mutations and their associations with the clinicopathological characteristics of primary $\mathrm{MM}$ showed an association of $B R A F$ mutations with younger age, location at the torso, non-chronically sun-damaged skin, SSM type, and advanced melanoma stage [16]. Similar results were identified in our sample set, but after using a logistic regression model, only ulceration and melanoma type were significant predictors.

In agreement with previous studies, $B R A F$ mutations were more commonly found in SSM, and with decreasing frequency in NM, ALM, and LMM [12, 13, 16, 17]. As expected, intraepidermal (in situ) MMs had an intermediate $B R A F$ mutation frequency. Upon further analysis, we identified that ulcerated NMs had a BRAF mutation frequency similar to SSMs $(56.4 \%)$, whereas non-ulcerated NMs had a percentage more akin to that of the ALM/LMM group (14.3\%). This could be explained if one considers ulcerated NMs as rapidly growing ex-SSMs, whose adjacent intraepidermal component was phased out [18].

Another histopathological parameter significantly related to $B R A F$ mutations was ulceration. Some studies have also reported increased $B R A F$ mutation percentage in ulcerated samples [12,17, 19-21], while others, including a meta-analysis, did not $[13,16]$. The 2 studies that included only 1 type 
Table 4. Histopathological Findings and BRAF Status of Primary MM

\begin{tabular}{|c|c|c|c|c|c|}
\hline & \multicolumn{2}{|c|}{ BRAF Status } & \multirow{2}{*}{ Total } & \multirow{2}{*}{ Mutated (\%) } & \multirow{2}{*}{$\mathbf{P}$} \\
\hline & WT & Mutated & & & \\
\hline \multicolumn{6}{|l|}{ Type $(\mathrm{n}=126)$} \\
\hline ALM & 7 & 1 & 8 & 12.5 & \multirow{6}{*}{0.004} \\
\hline LMM & 10 & 2 & 12 & 16.7 & \\
\hline Primary dermal & 2 & 1 & 3 & 33.3 & \\
\hline In situ & 9 & 5 & 14 & 35.7 & \\
\hline NM & 26 & 20 & 46 & 43.5 & \\
\hline SSM & 20 & 23 & 43 & 53.5 & \\
\hline Clark level $(\mathrm{n}=121)$ & $\begin{array}{c}2.68 \\
(2.4-3.0)\end{array}$ & $\begin{array}{c}2.54 \\
(2.3-2.8)\end{array}$ & $\begin{array}{c}2.62 \\
(2.4-2.8)\end{array}$ & & 0.741 \\
\hline 1 & 9 & 5 & 14 & 35.7 & \multirow{5}{*}{0.386} \\
\hline 2 & 31 & 23 & 54 & 42.6 & \\
\hline 3 & 12 & 13 & 25 & 52.0 & \\
\hline 4 & 12 & 8 & 20 & 40.0 & \\
\hline 5 & 7 & 1 & 8 & 12.5 & \\
\hline $\begin{array}{l}\text { Breslow thickness in } \mathrm{mm} \text {, } \\
(\mathrm{n}=113), \text { mean }(95 \% \mathrm{CI})\end{array}$ & $\begin{array}{c}3.63 \\
(2.8-4.5) \\
\end{array}$ & $\begin{array}{c}3.87 \\
(2.6-5.1) \\
\end{array}$ & $\begin{array}{c}3.73 \\
(4.9-7.8) \\
\end{array}$ & & 0.585 \\
\hline Breslow thickness $\leq 1 \mathrm{~mm}$ & 26 & 16 & 42 & 38.1 & \multirow{2}{*}{0.569} \\
\hline Breslow thickness $>1 \mathrm{~mm}$ & 46 & 36 & 82 & 43.9 & \\
\hline Tis & 10 & 6 & 16 & 37.5 & \multirow{5}{*}{0.828} \\
\hline $\mathrm{T} 1$ & 16 & 10 & 25 & 38.5 & \\
\hline $\mathrm{T} 2$ & 9 & 8 & 17 & 47.1 & \\
\hline T3 & 9 & 10 & 19 & 52.6 & \\
\hline $\mathrm{T} 4$ & 28 & 18 & 46 & 39.1 & \\
\hline \multicolumn{6}{|l|}{ TILs $(\mathrm{n}=109)$} \\
\hline Absent & 20 & 12 & 32 & 37.5 & \multirow{3}{*}{0.875} \\
\hline Nonbrisk & 32 & 24 & 56 & 42.9 & \\
\hline Brisk & 12 & 9 & 21 & 42.9 & \\
\hline $\begin{array}{l}\text { Mitoses }(n=129), \text { mean } \\
(95 \% \mathrm{CI})\end{array}$ & $\begin{array}{c}5.8 \\
(3.8-7.8) \\
\end{array}$ & $\begin{array}{c}6.74 \\
(4.6-8.8) \\
\end{array}$ & $\begin{array}{c}6.3 \\
(4.9-7.8) \\
\end{array}$ & & 0.307 \\
\hline Mitoses < 1/mm2 & 16 & 9 & 25 & 36.0 & \multirow{2}{*}{0.658} \\
\hline Mitoses $\geq 1 / \mathrm{mm} 2$ & 61 & 43 & 104 & 41.3 & \\
\hline \multicolumn{6}{|l|}{ Ulceration $(\mathrm{n}=129)$} \\
\hline No & 49 & 22 & 71 & 31.0 & \multirow{2}{*}{0.020} \\
\hline Yes & 28 & 30 & 58 & 51.7 & \\
\hline \multicolumn{6}{|l|}{ Solar elastosis $(n=129)$} \\
\hline No & 64 & 51 & 115 & 44.3 & \multirow{2}{*}{0.008} \\
\hline Yes & 13 & 1 & 14 & 7.1 & \\
\hline
\end{tabular}

$\mathrm{CI}=$ confidence interval; Tis = Tumor in situ; $\mathrm{WT}=$ wild type.

of melanoma (NM) identified a clear correlation [20, 21]. Furthermore, we identified increased mitotic rate in ulcerated melanomas (8.71 vs $2.21, \mathrm{P}<0.001$, Mann-Whitney test), yet no correlation between an increased mitotic rate and BRAF mutation percentage was identified (4.8 vs $5.6, \mathrm{P}$ $=0.307$, Mann-Whitney test $)$. This seems contradictory, since ulceration has been correlated with $B R A F$ mutations $[12,17$, 19] and increased mitotic rate [20,22]. Upon further analysis, lower mitotic rates were found in mutated tumors compared with wild-type ones (2.2 vs 6.83) in ALM, LMM, and nonulcerated NM, whereas the contrary was true concerning ulcerated NM and SSM (6.80 vs 4.82). 
$B R A F$ mutations have been previously linked to transition from radial to vertical growth phase $[6,23]$; to shorter recurrence-free, disease-specific survival [8]; and to overall survival with a more protracted growth rate [24]. However, the exact role and the pathways mirrored in specific morphological features such as ulceration and pathology types is elusive.

\section{Conclusions}

Our results suggest that $B R A F$ mutations are more frequent in SSM, ulcerated MMs independent of histological type, and MMs arising in non-chronically sun-damaged skin, ie, lacking solar elastosis. A significantly higher number of cases is required to clarify whether these correlations identified by many studies, as well as ours, are influenced by the variance of different populations, ages, and melanoma types included in each study. BRAF-mutated melanoma patients already have the advantage of being more effectively treated with drugs that specifically target $B R A F$-mutated cells, a fact that may mask the potential contribution of mutation presence to metastasis development. Furthermore, as both new immunerelated therapies and driver mutations for development and metastasis of MM are further identified, molecular profile of MMs may contribute to distinguishing melanoma types and estimating prognosis more accurately [25].

\section{Acknowledgment}

In loving memory of Professor Petros Karakitsos, a dedicated doctor, an outstanding scientist and a Pythagorean teacher, who passed away on June 26, 2017.

\section{References}

1. Siegel RL, Miller KD, Jemal A. Cancer statistics, 2017. CA Cancer J Clin. 2017;67(1):7-30.

2. Davies H, Bignell GR, Cox C, et al. Mutations of the BRAF gene in human cancer. Nature. 2002;417(6892):949-954.

3. Buscà R, Abbe P, Mantoux F, et al. Ras mediates the cAMPdependent activation of extracellular signal-regulated kinases (ERKs) in melanocytes. EMBO J. 2000;19(12):2900-2910.

4. Poynter JN, Elder JT, Fullen DR, et al. BRAF and NRAS mutations in melanoma and melanocytic nevi. Melanoma Res. 2006;16(4):267-273.

5. Arozarena I, Sanchez-Laorden B, Packer L, et al. Oncogenic BRAF induces melanoma cell invasion by downregulating the cGMPspecific phosphodiesterase PDE5A. Cancer Cell. 2011;19(1):4557.

6. Dong J, Phelps RG, Qiao R, et al. BRAF oncogenic mutations correlate with progression rather than initiation of human melanoma. Cancer Res. 2003;63(14): 3883-3885.

7. Shinozaki M, Fujimoto A, Morton DL, Hoon DSB. Incidence of BRAF oncogene mutation and clinical relevance for primary cutaneous melanomas. Clin Cancer Res. 2004;10(5):1753-1757.

8. Barbour AP, Tang YH, Armour N, et al. BRAF mutation status is an independent prognostic factor for resected stage IIIB and
IIIC melanoma: implications for melanoma staging and adjuvant therapy. Eur J Cancer. 2014;50(15):2668-2676.

9. Flaherty KT, Puzanov I, Kim KB, et al. Inhibition of mutated, activated BRAF in metastatic melanoma. N Engl J Med. 2010;363(9):809-819.

10. Balch CM, Soong S-J, Gershenwald JE, et al. Prognostic factors analysis of 17,600 melanoma patients: validation of the American Joint Committee on cancer melanoma staging system. J Clin Oncol. 2001;19(16):3622-3634.

11. Spathis A, Georgoulakis J, Foukas P, et al. KRAS and BRAF mutation analysis from liquid-based cytology brushings of colorectal carcinoma in comparison with formalin-fixed, paraffin-embedded tissue. Anticancer Res. 2010;30(6):1969-1975.

12. Ellerhorst JA, Greene VR, Ekmekcioglu S, et al. Clinical correlates of NRAS and BRAF mutations in primary human melanoma. Clin Cancer Res. 2011;17(2):229-235.

13. Jakob JA, Bassett RL Jr, Ng CS, et al., NRAS mutation status is an independent prognostic factor in metastatic melanoma. Cancer. 2012;118(16):4014-4023.

14. Colombino M, Capone M, Lissia A, et al. BRAF/NRAS mutation frequencies among primary tumors and metastases in patients with melanoma. J Clin Oncol. 2012;30(20):2522-2529.

15. Lee JH, Choi JW, Kim YS. Frequencies of BRAF and NRAS mutations are different in histological types and sites of origin of cutaneous melanoma: a meta-analysis. $\mathrm{Br} J$ Dermatol. 2011;164(4):776-784.

16. Kim SY, Kim SN, Hahn HJ, Lee YW, Choe YB, Ahn KJ. Metaanalysis of BRAF mutations and clinicopathologic characteristics in primary melanoma. J Am Acad Dermatol. 2015;72(6):10361046.e2.

17. Yamazaki N, Tanaka R, Tsutsumida A, et al. BRAF V600 mutations and pathological features in Japanese melanoma patients. Melanoma Res. 2015;25(1):9-14.

18. Mooi WJ, Krausz T. Melanoma: subtypes and variants. In: Hodder A, ed. Pathology of Melanocytic Disorders. London: Taylor \& Francis Ltd. 2007:285-342.

19. Safaee Ardekani G, Jafarnejad SM, Khosravi S, Martinka M, Ho V, Li G. Disease progression and patient survival are significantly influenced by BRAF protein expression in primary melanoma. $\mathrm{Br}$ J Dermatol. 2013;169(2):320-328.

20. Akslen LA, Angelini S, Straume O, et al. BRAF and NRAS mutations are frequent in nodular melanoma but are not associated with tumor cell proliferation or patient survival. I Invest Dermatol. 2005;125(2):312-317.

21. Hugdahl E, Kalvenes MB, Puntervoll HE, Ladstein RG, Akslen LA. BRAF-V600E expression in primary nodular melanoma is associated with aggressive tumour features and reduced survival. Br J Cancer. 2016;114(7):801-808.

22. Shen S, Wolfe R, McLean CA, Haskett M, Kelly JW. Characteristics and associations of high-mitotic-rate melanoma. JAMA Dermatol. 2014;150(10):1048-1055.

23. Greene VR, Johnson MM, Grimm EA, Ellerhorst JA. Frequencies of NRAS and BRAF mutations increase from the radial to the vertical growth phase in cutaneous melanoma. J Invest Dermatol. 2009;129(6):1483-1488.

24. Mar VJ, Liu W, Devitt B, et al. The role of BRAF mutations in primary melanoma growth rate and survival. $\mathrm{Br} J$ Dermatol. 2015;173(1):76-82.

25. Akbani R, Akdemir KC, Aksoy AA, et al; Cancer Genome Atlas Network. Genomic Classification of Cutaneous Melanoma. Cell. 2015;161(7):1681-1696. 